\title{
Non-left-orderable double branched coverings
}

\author{
TETSUYA ITO
}

\begin{abstract}
We develop a method to show the fundamental group of the double branched covering of links is not left-orderable by introducing a notion of coarse presentation. Like usual group presentations, a coarse presentation is given by a set of generators and relations, but inequalities are allowed as relations. By using coarse presentations, we give a family of links whose double branched covering has non-left-orderable fundamental group. Our family of links includes many known and new examples.
\end{abstract}

\section{Introduction}

A left ordering of a group $G$ is a total ordering $<_{G}$ of $G$ preserved by the left action of $G$. That is, $a<_{G} b$ implies $g a<_{G} g b$ for all $g, a, b \in G$. $G$ is called left-orderable if $G$ admits at least one left ordering. We adopt the convention that the trivial group $G=\{1\}$ is not left-orderable. As we will see in Section 5, this convention is natural when we study the relationship between left orderings and topology of 3-manifolds.

For a link $L$ in $S^{3}$, let $\Sigma_{2}(L)$ be the double branched covering of $L$. In this paper we study the non-existence of left orderings of $\pi_{1}\left(\Sigma_{2}(L)\right)$, the fundamental group of the double branched covering. The aim of this paper is to give a new method to show $\pi_{1}\left(\Sigma_{2}(L)\right)$ is not left-orderable, by introducing a coarse presentation.

A coarse presentation is a generalization of a group presentation. Like a usual group presentation, a coarse presentation is given by a set of generators and relations. The main differences are:

(1) A coarse presentation allows us to include inequalities as its relations.

(2) Unlike a group presentation, a coarse presentation does not determine the whole group structure: it rather provides partial information about group structure and left-orderings.

For a link diagram $D$ of a link $L$, we construct a coarse presentation, which we call the coarse Brunner's presentation, by generalizing a presentation of $\pi_{1}\left(\Sigma_{2}(L)\right)$ due to Brunner [3]. 
Using coarse Brunner's presentations, we will give various families of links whose double branched coverings have non-left-orderable fundamental group. In Theorem 4.1, we will treat links represented by diagrams similar to alternating link diagrams. This family of links contains alternating links, hence extends the result obtained in Boyer, Gordon and Watson [1] and Greene [8]: the double branched covering of an alternating link has non-left-orderable fundamental group. In Theorems 4.4 and 4.6, we will show that the double branched coverings of links represented by some particular diagrams have non-left-orderable fundamental group. These families of links contain positive knots of genus two and many non-alternating links.

The reader should regard these theorems rather as examples of coarse presentation arguments. In a similar manner, coarse Brunner's presentations allow us to find a lot of other examples of links whose branched double covering has non-left-orderable fundamental groups.

Although it is possible to prove all results in this paper by using usual group presentations (Brunner's presentation), our coarse presentation argument has several benefits. First of all, the coarse presentation is much simpler than the usual group presentation: it has fewer generators and fewer relations. In particular, by using coarse presentations, a proof of non-left-orderability becomes much simpler compared with a proof based on usual group presentations.

Moreover, in the proof of non-left-orderability, coarse Brunner's presentations allow us to separate the role of the link diagrams into the local properties and the global properties. That is, our proofs of non-left-orderability are valid if we replace a crossing with certain algebraic tangles. Thus a coarse presentation argument provides a more unified point of view in a proof of non-left-orderability.

The plan of this paper is as follows. In Section 2 we review standard notions of tangles and a presentation of $\pi_{1}\left(\Sigma_{2}(L)\right)$ due to Brunner [3]. We construct the coarse Brunner's presentation in Section 3, and give a non-left-orderability criterion based on coarse Brunner's presentation (Theorem 3.11). By using coarse Brunner's presentations, in Section 4 we will provide families of links whose double branched coverings have non-left-orderable fundamental group. In Section 5 we briefly discuss a relationship between the results in this paper and the $\mathrm{L}-$ space conjecture, a conjecture on orderability and Heegaard Floer homology.

\section{Acknowledgements}

The author would like to thank Joshua Greene and Liam Watson for comments on an earlier version of the paper. This research was partially supported by JSPS Research Fellowships for Young Scientists. 


\section{Presentation of the fundamental group of double branched cover}

\subsection{Algebraic and rational tangles}

First we review basic notions of tangles (see Kauffman and Lambropoulou [10] for fundamental facts on rational tangles).

Let $D$ be a diagram of a link $L$ in $S^{3}$ and consider the checker board coloring of $D$ chosen so that the color of the unbounded region is white. Let $A$ be a tangle that is a part of the diagram $D$. We define the east, west, south and north sides of $A$ as in Figure 1. In the rest of paper, we always assume that the west and east sides of a tangle $A$ are colored by black in the checker board coloring of $D$. We will often regard $A$ as an oriented arc from the east side to the west side. We call the north side of $A$ (resp. the south side of $A$ ) the left-adjacent region (resp. right-adjacent region) of $A$, and denote it by $R_{l}(A)$ (resp. $R_{r}(A)$ ). See Figure 1 .
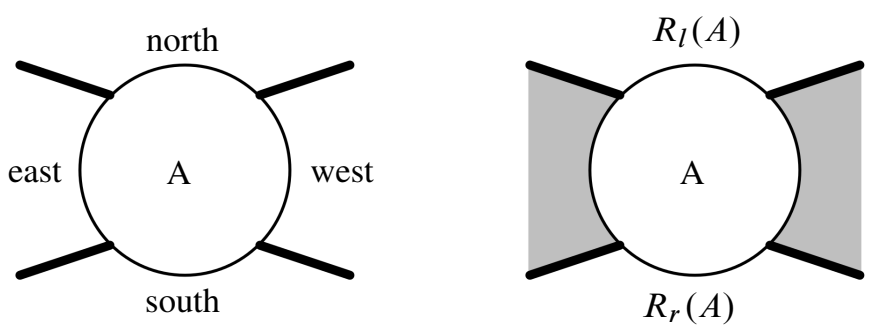

Figure 1: Adjacent regions of tangles

Under this coloring convention, the elementary tangles $[ \pm m]$ and $\left[ \pm \frac{1}{m}\right](m \in \mathbb{Z}-\{0\})$ are given by Figure 2. For two tangles $P$ and $Q$, the (tangle) sum $P+Q$ and the (tangle) product $P * Q$ are defined as in Figure 2 .

For a non-zero rational number $\frac{q}{p}$, let us take its continued fraction:

$$
\frac{q}{p}=a_{1}+\frac{1}{a_{2}+\frac{1}{a_{3}+\cdots}}
$$

A rational tangle $Q\left(\frac{q}{p}\right)$ is an algebraic tangle defined by

$$
Q\left(\frac{q}{p}\right)=\left(\cdots\left(\left(\left[a_{n}\right] *\left[\frac{1}{a_{n-1}}\right]\right)+\left[a_{n-2}\right]\right) * \cdots *\left[\frac{1}{a_{2}}\right]\right)+\left[a_{1}\right] .
$$




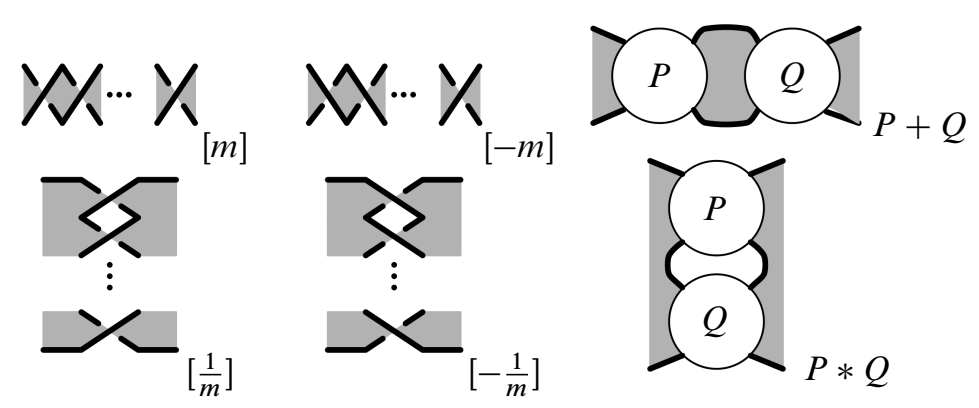

Figure 2: Elementary tangles and tangle sum, products

The isotopy class of the rational tangle $Q\left(\frac{q}{p}\right)$ does not depend on a choice of the continued fractions. In particular, for a non-zero integer $m$, the rational tangles $Q(m)$ and $Q\left(\frac{1}{m}\right)$ are isomorphic to the elementary tangles $[m]$ and $\left[\frac{1}{m}\right]$, respectively.

An algebraic tangle is a tangle obtained from rational tangles by repetitions of + and $*$ operations. Since rational tangles are obtained from elementary tangles by + and $*$ operations, an algebraic tangle is made of elementary tangles. In this paper we will use elementary tangles as a building block of algebraic tangles.

\subsection{Brunner's presentation}

In this section we review Brunner's presentation of the fundamental group of doublebranched coverings. For a checker board coloring of a diagram $D$, the regions colored by black define a (possibly non-orientable) compact surface that bounds $L$. We call this surface the checker board surface.

The checker board surface is decomposed as a union of discs and twisted bands in an obvious way. Among such decompositions, we choose the maximal one: the disctwisted band decomposition having the minimal number of twisted bands. Then we associate the labeled planar graph $G$, which we call the decomposition graph, and the oriented planar graph $\widetilde{G}$, which we call the connectivity graph, as follows.

The vertices of the decomposition graph $G$ are the discs in the maximal disc-twisted band decomposition. For a twisted band connecting two discs, we assign an edge of $G$ so that it connects corresponding vertices. The label of edge $e$ is a non-zero integer $i(e)$, the signed number of twistings, as defined in Figure 3. We call a component of $\mathbb{R}^{2}-G$ a region of the link diagram $D$. A region of $D$ is identified with a white-colored region of the diagram $D$.

The connectivity graph $\widetilde{G}$ is obtained from $G$ as follows. The vertices of $\widetilde{G}$ are the same as $G$. We connect two vertices by one edge if and only if there exists a twisted band 


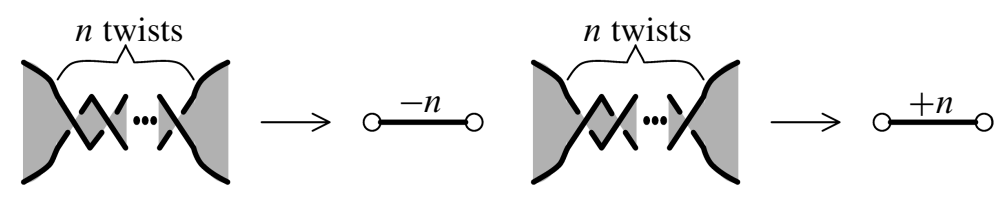

Figure 3: Labeled edge of the graph $G$

connecting two discs corresponding to the vertices. We choose an arbitrary orientation of edges and make $\widetilde{G}$ an oriented graph. We say an edge $w$ of $G$ corresponds to an edge $W$ of $\widetilde{G}$ if $w$ and $W$ connect the same vertices. An orientation of edges in $\widetilde{G}$ induces an orientation of corresponding edges of $G$, so we will always regard $G$ as an oriented graph once we have fixed the connectivity graph $\widetilde{G}$.

By using these notions, Brunner's presentation of $\pi_{1}\left(\Sigma_{2}(L)\right)$ is given as follows.

Theorem 2.1 (Brunner's presentation of $\pi_{1}\left(\Sigma_{2}(L)\right)$ [3]) Let $L$ be an unsplittable link in $S^{3}$ represented by a diagram $D$, and $G$ and $\widetilde{G}$ be the decomposition and the connectivity graphs. Then the fundamental group of $\Sigma_{2}(L)$ has the following presentation, which we call Brunner's presentation.

\section{[Generators]}

Edge generators $\left\{W_{i}\right\}$, the set of edges of the connectivity graph $\widetilde{G}$.

Region generators $\left\{R_{i}\right\}$, the set of regions of the link diagram $D$.

\section{[Relations]}

Local edge relations $W=\left(R_{l}(w)^{-1} R_{r}(w)\right)^{a}$, where $w$ is an edge of $G$ with label $a, R_{l}(w)$ and $R_{r}(w)$ are left-and right-adjacent regions of the edge $w$, and $W$ is an edge generator that corresponds to $w$.

Global cycle relations $W_{n}^{ \pm 1} \cdots W_{1}^{ \pm 1}=1$ if the edge-path $W_{n}^{ \pm 1} \cdots W_{1}^{ \pm 1}$ forms a loop in $\mathbb{R}^{2}$. Here $W_{i}^{-1}$ means the edge $W_{i}$ with the opposite orientation.

Vanishing relation $R_{0}=1$ where $R_{0}$ is the unbounded region.

Here we use the convention that the composite $W_{2} W_{1}$ represents the edge path that goes along $W_{1}$ first, then goes along $W_{2}$.

Example 1 Let $D$ be the knot diagram on the left in Figure 4. The decomposition graph $G$ and the connectivity graph $\widetilde{G}$ are given in Figure 4. Brunner's presentation 
of $\pi_{1}\left(\Sigma_{2}(L)\right)$ is given by

$$
\left\langle\begin{array}{c|lll}
A, B, C & \begin{array}{l}
W_{1}=A^{2} \\
W_{4}=\left(B^{-1} A\right)^{2}
\end{array} W_{5}=\left(B^{2}\right. & W_{3}=C \\
W_{1}, \ldots, W_{6} & W^{-1} & W_{6}=\left(C^{-1} A\right)^{-1} \\
W_{6} W_{4} W_{1}=1 & W_{4}^{-1} W_{5}^{-1} W_{2}=1 & W_{6}^{-1} W_{3}^{-1} W_{5}=1
\end{array}\right\rangle
$$

Here we used the vanishing relation to remove the trivial region generator.
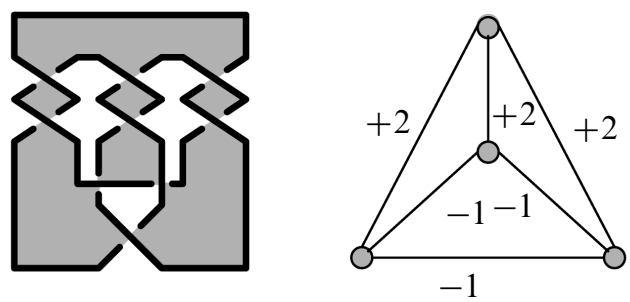

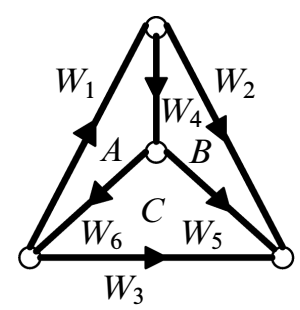

Figure 4: Example of Brunner's presentation

\section{Coarse Brunner's presentation}

In this section we construct the coarse Brunner's presentation, and give a non-leftorderability criterion (Theorem 3.11) based on the coarse Brunner's presentation.

Our construction of the coarse Brunner's presentation consists of three steps: First we introduce a tangle-strand decomposition and a coarse decomposition graph, which generalize a disc-band decomposition and a decomposition graph. Next we define a tangle element, which generalizes an edge generator in Brunner's presentation. We will observe that tangle elements satisfy relations that generalize the global cycle relations (Lemma 3.1). Finally, we introduce a generalization of the local edge relations, called coarse edge relations, which are given by inequalities.

\subsection{Coarse decomposition graph}

Recall that Brunner's presentation was constructed from a disc-band decomposition of the checker board surface of a link diagram $D$. In a diagrammatic point of view, a disc-band decomposition is regarded as a decomposition of $D$ into integer tangles and unknotted strands: integer tangles correspond to twisted bands, and the remaining black-colored regions correspond to discs.

To get a "coarse" presentation, we use a coarser decomposition of $D$. We consider a decomposition of $D$ as a union of algebraic tangles and unknotted strands. We call 
such a decomposition of $D$ a tangle-strand decomposition. A disc-band decomposition is a special case of the tangle-strand decomposition where all algebraic tangles are integer tangles.

As in a disc-band decomposition, a tangle-strand decomposition of $D$ defines the decomposition of the checker board surface as discs and subsurfaces corresponding to tangles. For a tangle-strand decomposition, we construct the coarse decomposition graph as follows. The vertex of $\Gamma$ is a disc part of the tangle-strand decomposition. For each algebraic tangle $A$, we assign an edge of $\Gamma$ having the label $A$ as in Figure 5, oriented from the east side of $A$ to the west side of $A$.

We call a connected component of $\mathbb{R}^{2}-\Gamma$ a region of $\Gamma$. By definition, a region of $\Gamma$ is naturally regarded as a region of $D$, that is, a connected component of $\mathbb{R}^{2}-G$, so it corresponds to a region generator of Brunner's presentation. By abuse of notation, we will regard a region $R$ of $\Gamma$ as an element of $\pi_{1}\left(\Sigma_{2}(L)\right)$ by considering the corresponding region generator in Brunner's presentation.

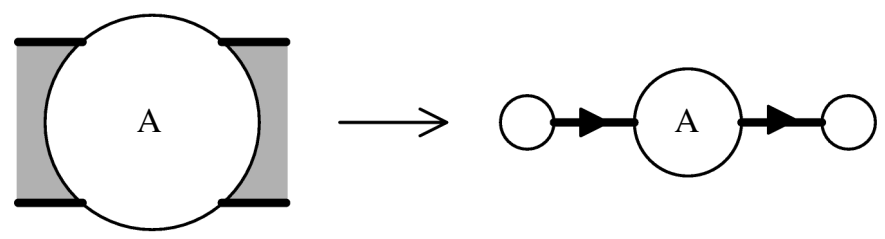

Figure 5: The coarse decomposition graph $\Gamma$

\subsection{Tangle elements: generators of the coarse Brunner's presentation}

In this section, we introduce a tangle element $W_{A} \in \pi_{1}\left(\Sigma_{2}(L)\right)$ for each tangle in a tangle-strand decomposition of $D$.

First of all, we define $W_{A}$ for elementary tangles. In the connectivity graph $G$, an elementary tangle $Q$ appears as an edge $W$. We define $W_{Q}=W \in \pi_{1}\left(\Sigma_{2}(L)\right)$, the corresponding edge generator $W$ in Brunner's presentation.

For a general algebraic tangle $A$, we inductively define $W_{A}$ as follows. Assume that $A$ is made of $n$ elementary tangles, so there exist algebraic tangles $A^{\prime}$ and $A^{\prime \prime}$ made of at most $(n-1)$ elementary tangles such that $A=A^{\prime}+A^{\prime \prime}$ or $A=A^{\prime} * A^{\prime \prime}$. Then we define $W_{A}$ by

$$
W_{A}= \begin{cases}W_{A^{\prime \prime}} W_{A^{\prime}} & \left(A=A^{\prime}+A^{\prime \prime}\right), \\ W_{A^{\prime}} & \left(A=A^{\prime} * A^{\prime \prime}\right) .\end{cases}
$$


There is an alternative definition of $W_{A}$ in terms of edge-path of the connectivity graph $\widetilde{G}$. Let $v$ and $v^{\prime}$ be the vertices of the connectivity graph $\widetilde{G}$ that correspond to the east and west sides of $A$, and let $\widetilde{G_{A}}$ be the subgraph of $\widetilde{G}$ derived from the sub-diagram $A$ of $D$. Then $W_{A}$ is equal to an element of $\pi_{1}\left(\Sigma_{2}(L)\right)$ that corresponds to the uppermost edge-path in $\widetilde{G_{A}}$ connecting $v$ and $v^{\prime}$, as explained in Figure 6 . This is why we regard $W_{A}$ as a generalization of edge generators.
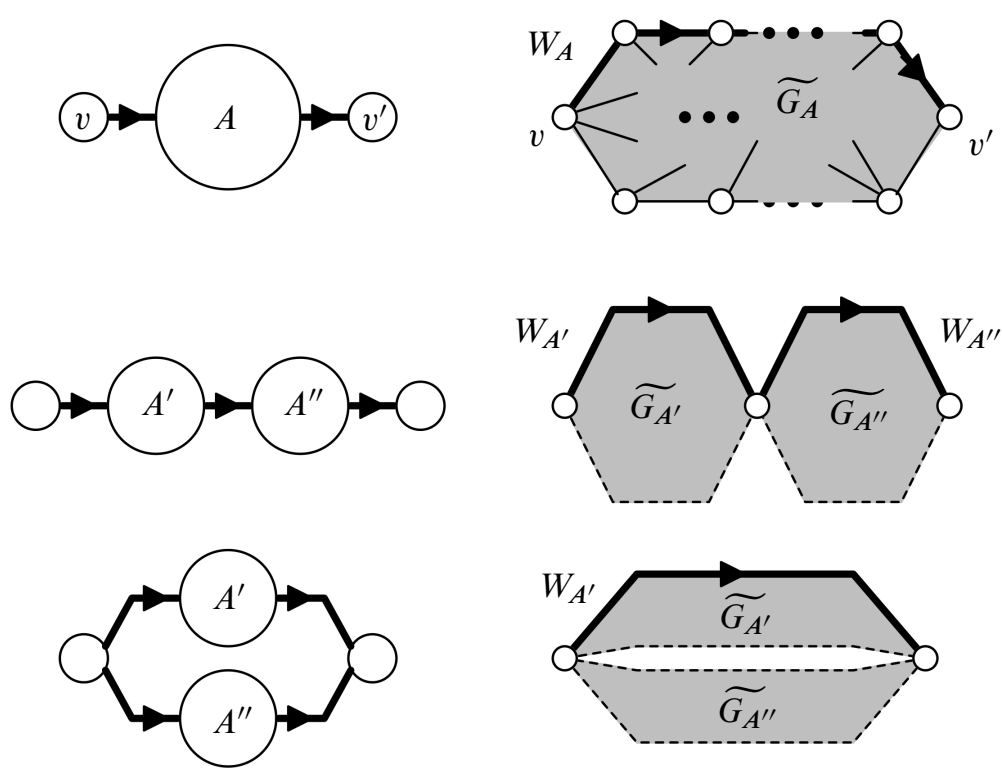

Figure 6: Tangle element as edge-path of $\widetilde{G}$

We observe the following generalization of global cycle relations.

Lemma 3.1 (Global cycle relation for tangle elements) Let $\Gamma$ be a coarse decomposition graph. For an algebraic tangle $A$ in a tangle-strand decomposition, let $E_{A}$ be an edge of the coarse decomposition graph $\Gamma$. If an edge-path $E_{A_{n}}^{ \pm 1} \cdots E_{A_{2}}^{ \pm 1} E_{A_{1}}^{ \pm 1}$ of $\Gamma$ forms a loop in $\mathbb{R}^{2}$, then the corresponding element satisfies

$$
W_{A_{n}}^{ \pm 1} \cdots W_{A_{2}}^{ \pm 1} W_{A_{1}}^{ \pm 1}=1 \text {. }
$$

Proof Assume that an edge-path

$$
E_{A_{n}}^{ \pm 1} \cdots E_{A_{2}}^{ \pm 1} E_{A_{1}}^{ \pm 1}
$$

of $\Gamma$ forms a loop in $\mathbb{R}^{2}$. Regarding each $E_{A_{i}}$ as an edge-path of $\widetilde{G}$, the edge-path $E_{A_{n}}^{ \pm 1} \cdots E_{A_{2}}^{ \pm 1} E_{A_{1}}^{ \pm 1}$, viewed as an edge-path of $\widetilde{G}$, forms a loop in $\mathbb{R}^{2}$ as well. Hence by the global cycle relations of Brunner's presentation, $W_{A_{n}}^{ \pm 1} \cdots W_{A_{2}}^{ \pm 1} W_{A_{1}}^{ \pm 1}=1$. 


\subsection{Universal range and coarse edge relation}

In this section we give a generalization of the local edge relations, which is the most important point of coarse Brunner's presentations.

For a left ordering $<_{G}$ of a group $G, X \in G$, and rational numbers $a=\frac{q}{p}, b=\frac{s}{r}(a \leq b)$, $p, q, r, s \in \mathbb{Z}, p, r>0$, let $\llbracket a, b \rrbracket_{X,<_{G}}$ be a subset of $G$ defined by

$$
\llbracket a, b \rrbracket_{X,<_{G}}= \begin{cases}\left\{g \in G \mid X^{q} \leq_{G} g^{p}, g^{r} \leq_{G} X^{s}, X g=g X\right\} & \text { if } X \geq_{G} 1, \\ \left\{g \in G \mid X^{s} \leq_{G} g^{r}, g^{p} \leq_{G} X^{q}, X g=g X\right\} & \text { if } X \leq_{G} 1 .\end{cases}
$$

We define

$$
\begin{aligned}
\llbracket\left[a,+\infty \rrbracket_{X,<_{G}}\right. & =\bigcup_{b>a} \llbracket\left[a, b \rrbracket_{X,<_{G}},\right. \\
\llbracket-\infty, b \rrbracket_{X,<_{G}} & =\bigcup_{b>a} \llbracket\left[a, b \rrbracket_{X,<_{G}},\right. \\
\llbracket-\infty,+\infty \rrbracket_{X,<_{G}} & =\bigcup_{a \in \mathbb{Z}_{>0}} \llbracket-a, a \rrbracket_{X,<_{G}} .
\end{aligned}
$$

If $g$ commutes with $X$, then $X^{m q} \leq_{G} g^{m p}(m>0)$ if and only if $X^{q} \leq_{G} g^{p}$. Hence $\llbracket a, b \rrbracket_{X,<_{G}}$ does not depend on a choice of the representatives of rationals $a=\frac{q}{p}$ and $b=\frac{s}{r}$ under the assumption $p, r>0$. For $a \in \mathbb{Q} \cup\{-\infty\}$ and $b \in \mathbb{Q} \cup\{+\infty\}(a \leq b)$, we define

$$
\llbracket a, b \rrbracket_{X}=\bigcap_{<_{G} \in L O(G)} \llbracket\left[a, b \rrbracket_{X,<_{G}}\right.
$$

where $L O(G)$ denotes the set of all left orderings of $G$. We say $\llbracket a, b \rrbracket$ is an $X-$ universal range of $g \in G$ if $g \in[a, b]_{X}$. By definition, if $X=1$ and $\left.g \in[-\infty,+\infty]\right]_{X}$, then $g=1$. This is an important feature of an $X$-universal range.

The followings are basic properties of universal ranges.

Lemma 3.2 Let $X, Y \in G, a, c \in \mathbb{Q} \cup\{-\infty\}$ and $b, d \in \mathbb{Q} \cup\{+\infty\}$ such that $a \leq b$ and $c \leq d$.

(1) If $[a, b] \subset[c, d]$ as a subset of $\mathbb{Q} \cup\{ \pm \infty\}$, then $\llbracket a, b \rrbracket_{X} \subset \llbracket\left[c, d \rrbracket_{X}\right.$ as a subset of $G$.

(2) Assume that $g \in \llbracket\left[a, b \rrbracket_{X}\right.$ and $h \in\left[\llbracket c, d \rrbracket_{X}\right.$.

(a) If $g h=h g$, or $a, b, c, d \in \mathbb{Z} \cup\{ \pm \infty\}$, then

$$
g h \in \llbracket a+c, b+d \rrbracket_{X} .
$$


Here we interpret

$$
\begin{cases}(+\infty)+r=r+(+\infty)=(+\infty), & r \in \mathbb{Q} \cup\{+\infty\}, \\ (-\infty)+r=(-\infty)+r=(-\infty), & r \in \mathbb{Q} \cup\{-\infty\} .\end{cases}
$$

(b) Assume that $0<a, c$, or $b, d<0$. If $X Y=Y X$, or $a, b, c, d \in 1 / \mathbb{Z}=$ $\left\{\frac{1}{n} \mid n \in \mathbb{Z}\right\} \cup\{ \pm \infty\}$, then

$$
g \in \llbracket\left(a^{-1}+c^{-1}\right)^{-1},\left(b^{-1}+d^{-1}\right)^{-1} \rrbracket_{X Y} .
$$

Here we interpret

$$
\begin{cases}(+\infty)^{-1}=(-\infty)^{-1}=0, & \\ 0^{-1}=(+\infty) & (\text { a }, c>0) \\ 0^{-1}=(-\infty) & (b, d<0)\end{cases}
$$

Proof Let $<_{G}$ be an arbitrary left ordering of $G$. (1) is obvious from the definition. To prove (2)(a), let us put $a=\frac{m}{N}, b=\frac{M}{N}, c=\frac{p}{Q}$ and $d=\frac{P}{Q}$, where $m, M, N, p, P$, $Q \in \mathbb{Z}$ and $N, Q>0$. We prove (2)(a) for the case $X \geq_{G} 1$. The case $X \leq_{G} 1$ is similar. Then by hypothesis,

$$
X^{m} \leq_{G} g^{N} \leq_{G} X^{M}, \quad X^{p} \leq_{G} h^{Q} \leq_{G} X^{P},
$$

hence

$$
X^{m Q+N p} \leq_{G} g^{N Q} h^{N Q} \leq_{G} X^{M Q+N P}
$$

Since we have assumed that either $N=Q=1$ or that $g$ and $h$ commute,

$$
X^{m Q+N p} \leq_{G}(g h)^{N Q} \leq_{G} X^{M Q+N P},
$$

which implies $g h \in \llbracket\left[a+c, b+d \rrbracket_{X,<_{G}}\right.$. Since $<_{G}$ is arbitrary, this shows $g h \in$ $\llbracket\left[a+c, b+d \rrbracket_{X}\right.$. The case that some of $a, b, c, d$ are $\pm \infty$ is proved in a similar way.

Finally, we prove (2)(b) for the case $a, c>0$ and $X \geq_{G} 1$. The other cases are proved in a similar way. Since $g \in \llbracket\left[a, b \rrbracket_{X}(a>0)\right.$, the assumption $X \geq_{G} 1$ implies $g \geq_{G} 1$. Then in turn, $g \in \llbracket[c, d]_{Y}(c>0)$ implies $Y \geq_{G} 1$, so $X Y \geq_{G} 1$.

First we treat the case $b, d \neq+\infty$. Let us put $a=\frac{m}{N}, b=\frac{M}{N}, c=\frac{p}{Q}$ and $d=\frac{P}{Q}$, where $m, M, N, p, P, Q \in \mathbb{Z}_{>0}$. Then we have inequalities

$$
X^{m} \leq_{G} g^{N} \leq_{G} X^{M}, \quad Y^{p} \leq_{G} g \leq^{P} .
$$

Hence we conclude

$$
X^{m p} Y^{m p} \leq_{G} g^{N p+m Q}, \quad g^{N P+M Q} \leq_{G} X^{M P} Y^{M P} .
$$


Since we have assumed $p=m=1$ or $X$ and $Y$ commute,

$$
(X Y)^{m p} \leq_{G} g^{N p+m Q}, \quad g^{N P+M Q} \leq_{G}(X Y)^{M P},
$$

that is, $g \in \llbracket\left(a^{-1}+c^{-1}\right)^{-1},\left(b^{-1}+d^{-1}\right)^{-1} \rrbracket_{X Y}$.

Next we treat the case $d=+\infty$. Let us put $a=\frac{m}{N}, b=\frac{M}{N}, c=\frac{p}{Q}$ as before. The proof of the lower bound

$$
(X Y)^{m p} \leq_{G} g^{N p+m Q}
$$

is the same. We show the upper bound. $g \in\left[b,+\infty \rrbracket_{Y}\right.$ implies $g \leq_{G} Y^{P}$ for sufficiently large integer $P>0$.

For $0 \leq i$,

$$
\begin{aligned}
\left(X^{-i} Y X^{i}\right)^{i N P}=X^{-i} Y^{i N P} X^{i} & \geq_{G} X^{-i} Y^{i N P} \\
& \geq_{G} X^{-i} g^{i N}=g^{i N} X^{-i} \\
& \geq_{G} g^{i N} X^{-i m} \\
& \geq_{G} g^{i N} g^{-i N}=1
\end{aligned}
$$

so

$$
\left(X^{i} Y X^{-i}\right) \geq_{G} 1 \quad(0 \leq i) .
$$

Hence

$$
(X Y)^{M}=X^{M}\left(X^{-(M-1)} Y X^{M-1}\right) \cdots\left(X^{-1} Y X\right) Y \geq_{G} X^{M} \geq_{G} g^{N} .
$$

Thus, $g \in \llbracket\left(a^{-1}+c^{-1}\right)^{-1},\left(b^{-1}+(+\infty)^{-1}\right)^{-1}=b \rrbracket_{X Y}$. The case $b=+\infty$ is similar.

Now we are ready to generalize a local edge relation. Let $A$ be an algebraic tangle in a tangle-strand decomposition of $D$, and let $R_{l}(A)$ and $R_{r}(A)$ be the left- and right-adjacent regions of $A$, regarded as an element of $\pi_{1}\left(\Sigma_{2}(L)\right)$.

Lemma 3.3 The tangle element $W_{A}$ commutes with $\left(R_{l}(A)^{-1} R_{r}(A)\right)$.

Proof Assume that $A$ is made of $n$ elementary tangles. We prove the lemma by induction on $n$.

If $A=Q(m)(m \in \mathbb{Z})$, then by local edge relation $W_{A}=\left(R_{l}(A)^{-1} R_{r}(A)\right)^{m}, W_{A}$ commutes with $\left(R_{l}(A)^{-1} R_{r}(A)\right)$. Assume that $A=Q\left( \pm \frac{1}{m}\right) \quad(m \in \mathbb{Z})$. Let us consider the subgraph of the decomposition graph $G$ derived from sub-diagram $A$, 
which consists of two vertices and $m$ edges labeled by \pm 1 . By local edge relations, we have equalities

$$
W_{A}=\left(R_{0}^{-1} R_{1}\right)^{ \pm 1}=\left(R_{1}^{-1} R_{2}\right)^{ \pm 1}=\cdots=\left(R_{m-1}^{-1} R_{m}\right)^{ \pm 1},
$$

where $R_{0}, \ldots, R_{m}$ are region generators taken as in Figure 7. Observe that $R_{0}=$ $R_{l}(A)$ and $R_{m}=R_{r}(A)$. Hence

$$
W_{A}^{ \pm m}=\left(R_{0}^{-1} R_{1}\right)\left(R_{1}^{-1} R_{2}\right) \cdots\left(R_{m-1}^{-1} R_{m}\right)=\left(R_{l}(A)^{-1} R_{r}(A)\right),
$$

so $W_{A}$ commutes with $\left(R_{l}(A)^{-1} R_{r}(A)\right)$.
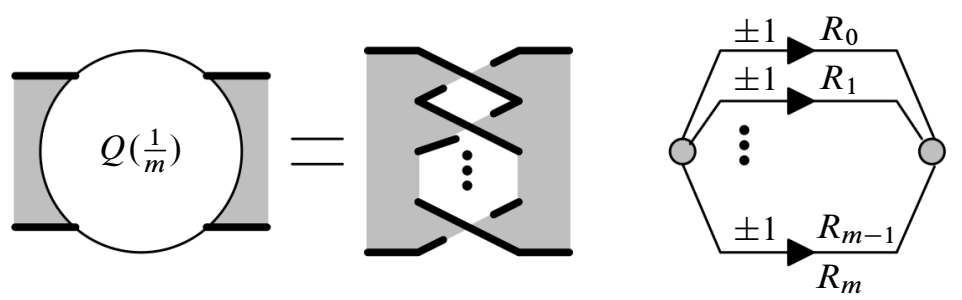

Figure 7: Subgraph of $G$ corresponds to an elementary tangle $Q\left(\frac{1}{m}\right)$

Now assume that $n>1$. There are algebraic tangles $A^{\prime}$ and $A^{\prime \prime}$ made of at most $(n-1)$ elementary tangles such that $A=A^{\prime}+A^{\prime \prime}$ or $A=A^{\prime} * A^{\prime \prime}$.

If $A=A^{\prime}+A^{\prime \prime}$, then $R_{l}(A)=R_{l}\left(A^{\prime}\right)=R_{l}\left(A^{\prime \prime}\right)$ and $R_{r}(A)=R_{r}\left(A^{\prime}\right)=R_{r}\left(A^{\prime \prime}\right)$. By induction, both $W_{A^{\prime}}$ and $W_{A^{\prime \prime}}$ commute with $\left(R_{l}(A)^{-1} R_{r}(A)\right)$, hence $W_{A}=W_{A^{\prime \prime}} W_{A^{\prime}}$ commutes with $\left(R_{l}(A)^{-1} R_{r}(A)\right)$.

If $A=A^{\prime} * A^{\prime \prime}$, then $R_{l}(A)=R_{l}\left(A^{\prime}\right), R_{r}\left(A^{\prime}\right)=R_{l}\left(A^{\prime \prime}\right)$ and $R_{r}\left(A^{\prime \prime}\right)=R_{r}(A)$. By induction, $W_{A^{\prime}}$ commutes with $\left(R_{l}^{-1}\left(A^{\prime}\right) R_{r}\left(A^{\prime}\right)\right)$ and $W_{A^{\prime \prime}}$ commutes with

$$
\left(R_{l}^{-1}\left(A^{\prime \prime}\right) R_{r}\left(A^{\prime \prime}\right)\right) \text {. }
$$

On the other hand, the global cycle relation shows $W_{A}=W_{A^{\prime}}=W_{A^{\prime \prime}}$. Hence we conclude $W_{A}$ commutes with

$$
\left(R_{l}^{-1}(A) R_{r}(A)\right)=\left(R_{l}\left(A^{\prime}\right)^{-1} R_{r}\left(A^{\prime}\right)\right)\left(R_{l}\left(A^{\prime \prime}\right)^{-1} R_{r}\left(A^{\prime \prime}\right)\right) .
$$

Based on this observation, we define a universal range of an algebraic tangle as follows.

Definition 3.4 A universal range of $A$ is an $\left(R_{l}(A)^{-1} R_{r}(A)\right)$-universal range of $W_{A}$. For $a, b \in \mathbb{Q} \cup\{ \pm \infty\}(a \leq b)$, we denote $A \in \llbracket a, b \rrbracket$ if $\llbracket a, b \rrbracket$ is an $\left(R_{l}(A)^{-1} R_{r}(A)\right)$ universal range of $W_{A}$. We say an algebraic tangle is bounded if $A \in[-\infty,+\infty \rrbracket$. 
By applying Lemma 3.2, we are able to compute a universal range of an algebraic tangle $A$ in many cases.

Proposition 3.5 (Universal ranges of algebraic tangles) Let $D$ be a link diagram and $A$ be an algebraic tangle in a tangle-strand decomposition of $D$.

(1) Assume that $A$ is an elementary tangle. If $A=Q(m)$, then $A \in \llbracket m, m \rrbracket$ and if $A=Q\left(\frac{1}{m}\right)$, then $A \in \llbracket \frac{1}{m}, \frac{1}{m} \rrbracket$.

(2) Let $A_{1}$ and $A_{2}$ be algebraic tangles and assume that $A_{1} \in \llbracket a, b \rrbracket$, and $A_{2} \in$ $\llbracket c, d \rrbracket$, where $a, c \in \mathbb{Q} \cup\{-\infty\}$ and $b, d \in \mathbb{Q} \cup\{+\infty\}$.

(a) Assume that $A=A_{1}+A_{2}$. If $a, b, c, d \in \mathbb{Z} \cup\{ \pm \infty\}$, or $W_{A_{1}}$ and $W_{A_{2}}$ commute, then

$$
A \in \llbracket a+c, b+d \rrbracket .
$$

(b) Assume that $A=A_{1} * A_{2}$ and that $0<a, c$, or $b, d<0$.

If $a, b, c, d \in 1 / \mathbb{Z}=\left\{\frac{1}{n} \mid n \in \mathbb{Z}-\{0\}\right\} \cup\{ \pm \infty\}$, or $R_{l}\left(A_{1}\right)^{-1} R_{r}\left(A_{1}\right)$ and $R_{l}\left(A_{2}\right)^{-1} R_{r}\left(A_{2}\right)$ commute, then

$$
A \in\left[\left(a^{-1}+c^{-1}\right)^{-1},\left(b^{-1}+d^{-1}\right)^{-1} \rrbracket .\right.
$$

Proof (1) was proved in Lemma 3.3, since we have seen that

$$
W_{A}=\left(R_{l}(A)^{-1} R_{r}(A)\right)^{m}
$$

if $A=Q(m)$, and that $W_{A}^{m}=\left(R_{l}(A)^{-1} R_{r}(A)\right)$ if $A=Q\left(\frac{1}{m}\right)$. (2) is nothing but a restatement of Lemma 3.2.

Corollary 3.6 If $Q=Q\left(\frac{q}{p}\right)$ is a rational tangle, then $W_{Q} \in \llbracket \frac{q}{p}, \frac{q}{p} \rrbracket$. That is,

$$
W_{Q}^{p}=\left(R_{l}(Q)^{-1} R_{r}(Q)\right)^{q} .
$$

Proof Let us write $Q\left(\frac{q}{p}\right)$ as

$$
Q\left(\frac{q}{p}\right)=\left(\cdots\left(\left(\left[a_{2 n+1}\right] *\left[\frac{1}{a_{2 n}}\right]\right)+\left[a_{n-2}\right]\right) * \cdots *\left[\frac{1}{a_{2}}\right]\right)+\left[a_{1}\right]
$$

where $a_{1}, \ldots, a_{2 n+1}$ is determined by the continued fraction:

$$
\frac{q}{p}=a_{1}+\frac{1}{a_{2}+\frac{1}{a_{3}+\cdots}}
$$

We prove the corollary by induction on $n$. The case $n=0$ is trivial. 
First assume that $n=1$, so $Q\left(\frac{q}{p}\right)=\left(\left[a_{3}\right] *\left[\frac{1}{a_{2}}\right]\right)+\left[a_{1}\right] .\left(R_{l}\left(\left[a_{3}\right]\right)^{-1} R_{r}\left(\left[a_{3}\right]\right)\right)$ commutes with $W_{\left[a_{3}\right]}$. Since

$$
W_{\left[a_{3}\right]}^{a_{2}}=W_{\left[\frac{1}{a_{2}}\right]}^{a_{2}}=\left(R_{l}\left(\left[\frac{1}{a_{2}}\right]\right)^{-1} R_{r}\left(\left[\frac{1}{a_{2}}\right]\right)\right),
$$

we conclude $\left(R_{l}\left(\left[a_{3}\right]\right)^{-1} R_{r}\left(\left[a_{3}\right]\right)\right)$ commutes with $\left(R_{l}\left(\left[\frac{1}{a_{2}}\right]\right)^{-1} R_{r}\left(\left[\frac{1}{a_{2}}\right]\right)\right)$. So by Proposition 3.5,

$$
\left(\left[a_{3}\right] *\left[\frac{1}{a_{2}}\right]\right)+\left[a_{1}\right] \in \llbracket \frac{q}{p}, \frac{q}{p} \rrbracket .
$$

To prove the case $n>1$, let us write

$$
Q\left(\frac{q}{p}\right)=\left(Q^{\prime} *\left[\frac{1}{a_{2}}\right]\right)+\left[a_{1}\right]
$$

where $Q^{\prime}$ is a rational tangle

$$
Q^{\prime}=Q\left(\frac{q^{\prime}}{p^{\prime}}\right)=\left(\cdots\left(\left(\left[a_{2 n+1}\right] *\left[\frac{1}{a_{2 n}}\right]\right)+\left[a_{n-2}\right]\right) * \cdots *\left[\frac{1}{a_{4}}\right]\right)+\left[a_{3}\right] .
$$

Then $\left(R_{l}\left(Q^{\prime}\right)^{-1} R_{r}\left(Q^{\prime}\right)\right)$ commutes with $W_{Q^{\prime}}$. Since $W_{Q^{\prime}}=W_{\left[\frac{1}{a_{2}}\right]}$,

$$
W_{Q^{\prime}}^{a_{2}}=W_{\left[\frac{1}{a_{2}}\right]}^{a_{2}}=R_{l}\left(\left[\frac{1}{a_{2}}\right]\right)^{-1} R_{r}\left(\left[\frac{1}{a_{2}}\right]\right) .
$$

So $\left(R_{l}\left(Q^{\prime}\right)^{-1} R_{r}\left(Q^{\prime}\right)\right)$ commutes with $\left(R_{l}\left(\left[\frac{1}{a_{2}}\right]\right)^{-1} R_{r}\left(\left[\frac{1}{a_{2}}\right]\right)\right)$. By induction, $Q^{\prime} \in$ $\llbracket \frac{q^{\prime}}{p^{\prime}}, \frac{q^{\prime}}{p^{\prime}} \rrbracket$. Thus by Proposition 3.5(2),

$$
Q=\left(Q^{\prime} *\left[\frac{1}{a_{2}}\right]\right)+\left[a_{1}\right] \in \llbracket \frac{q}{p}, \frac{q}{p} \rrbracket .
$$

A universal range obtained from Proposition 3.5 might not be optimal, although in most cases a universal range computed by Proposition 3.5 is sufficient to apply our theorems described in next section.

In some cases, with some additional assumptions or more careful arguments, we will often be able to compute a universal range even if one cannot apply Proposition 3.5, or will be able to get a better universal range.

Example 2 Let us consider the algebraic tangle $A=A_{1}+A_{2}=Q\left(\frac{1}{3}\right)+Q\left(\frac{1}{4}\right)$. We do not know whether $W_{A_{1}}$ commutes with $W_{A_{2}}$ or not. So according to Proposition 3.5, $A \in[0,2]$ since $Q\left(\frac{1}{3}\right) \in\left[0,1 \rrbracket\right.$ and $Q\left(\frac{1}{4}\right) \in[0,1]$. However, in this case we can get a better universal range as follows. 
Let $X=\left(R_{l}(A)^{-1} R_{r}(A)\right), W_{1}=W_{A_{1}}$ and $W_{2}=W_{A_{2}}$. Then $W_{1}^{3}=W_{2}^{4}=X$. Let $<_{G}$ be a left-ordering of $\pi_{1}\left(\Sigma_{2}(L)\right)$. We consider the case $1 \leq_{G} X$. The case $1 \geq_{G} X$ is treated in a similar way. Now

$$
W_{A}^{3}=\left(W_{1} W_{2}\right)^{3}=W_{1}^{3}\left(W_{1}^{-2} W_{2} W_{1}^{2}\right)\left(W_{1}^{-1} W_{2} W_{1}\right) W_{2} .
$$

Since $\left(W_{1}^{-i} W_{2} W_{1}^{i}\right)^{4}=X, W_{1}^{-i} W_{2} W_{1}^{i} \geq 1$ holds for all $i$. Thus, we get

$$
W_{A}^{3}=\left(W_{1} W_{2}\right)^{3} \geq_{G} W_{1}^{3}=X .
$$

Similarly, we have

$$
W_{A}^{4}=\left(W_{1} W_{2}\right)^{4}=W_{1} W_{2}^{4}\left(W_{2}^{-3} W_{1} W_{2}^{3}\right)\left(W_{2}^{-2} W_{1} W_{2}^{2}\right)\left(W_{2}^{-1} W_{1} W_{2}\right) .
$$

Since $\left(W_{2}^{-i} W_{1} W_{2}^{i}\right)^{3}=X, W_{2}^{-i} W_{1} W_{2}^{i} \leq_{G} X$ for all $i$. Thus, we get

$$
W_{A}^{4}=\left(W_{1} W_{2}\right)^{4} \leq_{G} X^{5} .
$$

Thus $\left.A \in\left[\frac{1}{3}, \frac{5}{4}\right]\right]$.

Next we compute a universal range of an algebraic tangle $B=A * A_{3}$ where $A_{3}=$ $Q\left(-\frac{1}{4}\right)$. Observe that one cannot apply Proposition 3.5(2)(b). However, in this situation we are able to compute universal range as follows.

Let us put $W_{3}=W_{A_{3}}$ and $Y=R_{l}\left(A_{3}\right)^{-1} R_{r}\left(A_{3}\right)$, so $X Y=R_{l}(B)^{-1} R_{r}(B)$. By local edge relation, $W_{3}^{-4}=Y$ and $W_{B}=W_{A}=W_{A_{3}}$. In particular, $Y$ commutes with $X$. Since we observed

$$
\begin{gathered}
X \leq_{G} W_{A}^{3}=W_{B}^{3} \leq_{G} W_{A}^{4}=W_{B}^{4} \leq_{G} X^{5} \\
(X Y)=X W_{B}^{-4} \leq_{G} W_{B}^{-1}, W_{B}^{-16}=W_{B}^{4} W_{B}^{-20} \leq_{G} X^{5} Y^{5}=(X Y)^{5} .
\end{gathered}
$$

Hence $B \in \llbracket-1,-\frac{5}{16} \rrbracket$.

\subsection{Coarse Brunner's presentations}

Now we are ready to give coarse Brunner's presentations. Let $D$ be a link diagram. We say a tangle-strand decomposition is nice if all tangles are bounded, that is, all tangles have universal range $A \in[-\infty,+\infty]$. For a nice tangle-strand decomposition let $\Gamma$ be the coarse decomposition graph. Summarizing all relations obtained so far, we get the following family of information about left-orderings and the group structure of $\pi_{1}\left(\Sigma_{2}(L)\right)$.

Definition 3.7 The coarse Brunner's presentation $\mathcal{C B}$ associated to a nice tanglestrand decomposition of a link diagram $D$ is a set of generators and relations given as follows. 


\section{[Generators]}

Tangle generator $\left\{W_{A}\right\}$, the set of tangle elements (the set of edges of $\Gamma$ ).

Region generator $\left\{R_{i}\right\}$, the set of connected components of $\mathbb{R}^{2}-\Gamma$ (the set of regions of $\Gamma$ ).

\section{[Relations]}

Local coarse relation $W_{A} \in \llbracket\left[m_{A}, M_{A} \rrbracket_{\left(R_{l}(A)^{-1} R_{r}(A)\right)}\right.$, where $\llbracket m_{A}, M_{A} \rrbracket$ is a universal range of $A$.

Global cycle relation $W_{n}^{ \pm 1} \cdots W_{1}^{ \pm 1}=1$ if the edge-path $W_{n}^{ \pm 1} \cdots W_{1}^{ \pm 1}$ of $\Gamma$ forms a loop in $\mathbb{R}^{2}$. Here $W_{i}^{-1}$ represents the path $W_{i}$ with the opposite orientation.

Vanishing relation $R_{0}=1$ where $R_{0}$ corresponds to the unbounded region.

Example 3 Let us consider a tangle-strand decomposition of a link diagram on the left in Figure 8, and assume that all tangles are bounded. The coarse decomposition graph $\Gamma$ is given on the right in Figure 8 . Let $\llbracket m_{i}, M_{i} \rrbracket$ be the universal range of $A_{i}$. Then the coarse Brunner's presentation associated to the tangle-strand decomposition is given as follows:

[Generators]

$$
W_{1}, W_{2}, \ldots, W_{6}, A, B, C
$$

\section{[Local coarse relations]}

$$
\begin{array}{ll}
W_{1} \in\left[m_{1}, M_{1} \rrbracket_{A}\right. & \left.W_{2} \in \llbracket m_{2}, M_{2} \rrbracket\right]_{B} \\
W_{3} \in \llbracket m_{3}, M_{3} \rrbracket_{C^{-1}} & W_{4} \in \llbracket m_{4}, M_{4} \rrbracket_{B^{-1} A} \\
W_{5} \in \llbracket\left[m_{5}, M_{5} \rrbracket_{B^{-1} C}\right. & W_{6} \in \llbracket m_{6}, M_{6} \rrbracket_{C^{-1} A}
\end{array}
$$

[Global cycle relations]

$$
W_{6} W_{4} W_{1}=1, \quad W_{4}^{-1} W_{5}^{-1} W_{2}=1, \quad W_{6}^{-1} W_{3}^{-1} W_{5}=1
$$

Here we put $W_{A_{i}}=W_{i}$, and simplified relations by removing the trivial region generator.

To use coarse Brunner's presentations to prove non-left-orderability, we need to add one additional restriction.

First of all, we introduce a notion of positive definite tangles. An algebraic tangle $A$ is called positive definite (resp. negative definite) if $A \in \bigcup_{a \in \mathbb{Q}>0} \llbracket a,+\infty \rrbracket$ (resp. $A \in \bigcup_{a \in \mathbb{Q}<0} \llbracket[-\infty, a \rrbracket)$. 

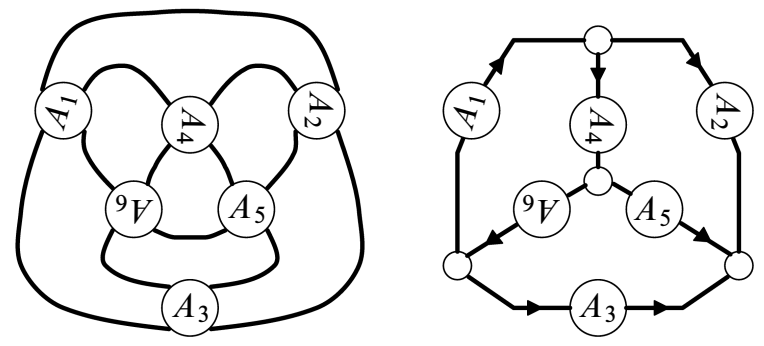

Figure 8: Example of coarse Brunner's presentation

A positive definite tangle can be seen as a generalization of positive crossing in a coarse presentation point of view. Recall that for a left-ordering $<_{G}$ of $\pi_{1}\left(\Sigma_{2}(L)\right)$ and an edge generator $W$ corresponding to positive twisted bands (positive crossings), by local edge relation, $W>_{G} 1$ if and only if $R_{l}(W)^{-1} R_{r}(W)>_{G} 1$. A positive definite algebraic tangle has the same property:

Lemma 3.8 For a left-ordering $<_{G}$ of $\pi_{1}\left(\Sigma_{2}(L)\right)$ and the tangle element $W_{A}$ of a positive definite tangle $A, W_{A}>_{G} 1$ if and only if $R_{l}(A)^{-1} R_{r}(A)>_{G} 1$.

Proof Let us put $X=R_{l}(A)^{-1} R_{r}(A)$. Since $A$ is positive definite, there exist rationals $0<\frac{m}{N} \leq \frac{M}{N}\left(m, M, N \in \mathbb{Z}_{>0}\right)$ such that $W_{A} \in \llbracket \frac{m}{N}, \frac{M}{N} \rrbracket_{X}$. If $X>_{G} 1$, then

$$
1<_{G} X^{m} \leq_{G} W_{A}^{N}
$$

so $W_{A}>_{G} 1$. Conversely, assume $W_{A}>_{G} 1$. If $X \leq_{G} 1$, then

$$
X^{M} \leq_{G} W_{A}^{N} \leq_{G} 1
$$

which is a contradiction, so $X>_{G} 1$.

Definition 3.9 An algebraic tangle $A$ is called special if it is bounded and belongs to the subset $\mathcal{S} \mathcal{A}$ of algebraic tangles that is inductively defined as follows:

(1) An elementary tangle belongs to $\mathcal{S} \mathcal{A}$.

(2) If $A, A^{\prime} \in \mathcal{S} \mathcal{A}$, then $A+A^{\prime} \in \mathcal{S} \mathcal{A}$.

(3) If $A, A^{\prime} \in \mathcal{S} \mathcal{A}$, and if either $A$ or $A^{\prime}$ is positive or negative definite, then $A * A^{\prime} \in \mathcal{S} \mathcal{A}$.

A special tangle-strand decomposition is a tangle-strand decomposition such that all tangles are special. 
Remark 1 There are many special algebraic tangles. For example, a bounded tangle obtained by applying Proposition 3.5 is special. In particular, rational tangles are special.

By Proposition 3.5, an alternating algebraic tangle is special, and positive or negative definite. The converse is not true. Let us consider an algebraic tangle $C=B+Q(2)$, where $B$ is the algebraic tangle given in Example 2. Then $C$ is non-alternating, but $C \in\left[1, \frac{27}{16} \rrbracket\right]$ so it is positive definite.

An important feature of special algebraic tangles is the following.

Lemma 3.10 Assume that $\pi_{1}\left(\Sigma_{2}(L)\right)$ is left-orderable. Let $A$ be a special algebraic tangle in a tangle-strand decomposition. Let $G_{A}$ and $\widetilde{G_{A}}$ be the subgraph of the decomposition and connectivity graph that correspond to the sub-diagram $A$. If $R_{r}(A)=R_{l}(A)=W_{A}=1$, then all edge and region generators of Brunner's presentation that appear in the subgraphs $G_{A}$ and $\widetilde{G_{A}}$ are equal to 1 .

Proof Assume that $A$ is made of $n$ elementary tangles. We prove by induction on $n$. First of all, assume that $A$ is an elementary tangle. If $A=Q(m)$, we have nothing to prove. If $A=Q\left( \pm \frac{1}{m}\right)$, then by local edge relations, we have equalities

$$
W_{A}=\left(R_{0}^{-1} R_{1}\right)^{ \pm 1}=\left(R_{1}^{-1} R_{2}\right)^{ \pm 1}=\cdots=\left(R_{m-1}^{-1} R_{m}\right)^{ \pm 1}
$$

where $R_{0}, \ldots, R_{m}$ are region generators taken as in Figure 7. By hypothesis, $W_{A}=$ $R_{0}=R_{m}=1$, so we conclude $R_{i}=0$ for all $i$.

Now we assume that $A=A^{\prime}+A^{\prime \prime}$ or $A=A^{\prime} * A^{\prime \prime}$ where $A^{\prime}$ and $A^{\prime \prime}$ are special algebraic tangles made of at most $(n-1)$ elementary tangles.

Assume that $A=A^{\prime}+A^{\prime \prime}$. By assumption,

$$
\left(R_{l}\left(A^{\prime}\right)^{-1} R_{r}\left(A^{\prime}\right)\right)=\left(R_{l}\left(A^{\prime \prime}\right)^{-1} R_{r}\left(A^{\prime \prime}\right)\right)=\left(R_{l}(A)^{-1} R_{r}(A)\right)=1 .
$$

Since $A^{\prime}$ and $A^{\prime \prime}$ are bounded, $W_{A^{\prime}}, W_{A^{\prime \prime}} \in \llbracket-\infty,+\infty \rrbracket_{1}$, which implies $W_{A^{\prime}}=$ $W_{A^{\prime \prime}}=1$. By inductive hypothesis, all region and edge generators in $G_{A}$ and $\widetilde{G_{A}}$ are trivial.

Next assume that $A=A^{\prime} * A^{\prime \prime}$. By hypothesis,

$$
R_{l}\left(A^{\prime}\right)=R_{l}(A)=R_{r}\left(A^{\prime \prime}\right)=R_{r}(A)=W_{A}=W_{A^{\prime}}=W_{A^{\prime \prime}}=1 .
$$

By definition of special algebraic tangles, $A^{\prime}$ or $A^{\prime \prime}$ is positive or negative definite. We treat the case where $A^{\prime}$ is positive definite. The other cases are similar. 
By Lemma 3.8, $W_{A^{\prime}}=1$ implies that $\left(R_{l}\left(A^{\prime}\right)^{-1} R_{r}\left(A^{\prime}\right)\right)=R_{r}\left(A^{\prime}\right)=1$. Thus $W_{A^{\prime}}=W_{A^{\prime \prime}}=R_{l}\left(A^{\prime}\right)=R_{r}\left(A^{\prime}\right)=R_{l}\left(A^{\prime \prime}\right)=R_{r}\left(A^{\prime \prime}\right)=1$, hence by induction all region and edge generators in $G_{A}$ and $\widetilde{G_{A}}$ are trivial.

Now we are ready to give a non-left-orderability criterion based on coarse Brunner's presentations. The next theorem says that to prove that $\pi_{1}\left(\Sigma_{2}(L)\right)$ is not left-orderable, it is sufficient to show that all region generators in the coarse Brunner's presentation are trivial.

Theorem 3.11 (Non-left-orderability criterion) Let $\mathcal{C B}$ be the coarse Brunner's presentation associated to a special tangle-strand decomposition of $D$. If $\pi_{1}\left(\Sigma_{2}(L)\right)$ is left-orderable, then there exists a region generator $R$ of $\mathcal{C B}$ such that $R \neq 1$.

Proof Assume that all region generators of $\mathcal{C B}$ are trivial. All tangles are bounded, so $\left.W_{A} \in[-\infty,+\infty]\right]_{1}$ hence $W_{A}=1$. Since all tangles are special, by Lemma 3.10 all region generators and edge generators in Brunner's presentations are trivial. Hence all generators of Brunner's presentation are trivial, so $\pi_{1}\left(\Sigma_{2}(L)\right)=1$. Since we adopted the convention that the trivial group is not left-orderable, this contradicts the hypothesis. (In fact, if $L$ is not the unlink, it is known that $\pi_{1}\left(\Sigma_{2}(L)\right) \neq 1$, so this cannot occur).

\section{Non-left-orderable double branched covering}

In this section we use coarse Brunner's presentations to show that the double branched covering of links represented by certain diagrams has non-left-orderable fundamental group. As we have already mentioned, the reader might regard the proof of these theorems rather as examples of how to show non-left-orderability via coarse Brunner's presentations. The arguments appearing in the following proofs are typical examples of how one may deduce a contradiction assuming left-orderability of $\pi_{1}\left(\Sigma_{2}(L)\right)$.

\subsection{Diagrams that are close to alternating diagrams}

The first example we treat is a link similar to an alternating link from the coarse presentation point of view. The proof of the next theorem is inspired by an argument of Greene in [8], and is regarded as an adaptation of Greene's argument for coarse Brunner's presentation.

Theorem 4.1 Let $D$ be a link diagram that admits a special tangle-strand decomposition such that all tangles are positive definite, or all tangles are negative definite. Then $\pi_{1}\left(\Sigma_{2}(L)\right)$ is not left-orderable. 
Proof Assume that $\pi_{1}\left(\Sigma_{2}(L)\right)$ has a left ordering $<_{G}$. We prove the case when all tangles are positive definite. The negative definite case is proved in a similar way. Let $\mathcal{C B}$ be the coarse Brunner's presentation.

By Theorem 3.11 there are at least two (non-trivial) distinct region generators. Let $R$ be the region generator that is a $<_{G}$-maximal among all region generators. With no loss of generality, we may assume $R>_{G} 1$, hence $R$ is a bounded region. Since we have assumed that there are at least two distinct region generators, we may choose $R$ so that there is a region generator $R^{\prime}<_{G} R$ that is adjacent to $R$.

Let us consider the global cycle relation $W_{1}^{ \pm 1} \cdots W_{k}^{ \pm 1}=1$ given by the edge-path representing $\partial R$, where $W_{i}$ denotes the tangle element of $A_{i}$. Since the property that an algebraic tangle $A$ is positive definite is independent of the choice of the orientations of edges in $\Gamma$, we may choose the tangle-strand decomposition (orientation of edge) so that the global cycle relation is given as $W_{1} \cdots W_{k}=1$. We choose orientations of the edges so that $R_{l}\left(A_{i}\right)=R$ holds for all $i$.

$R_{l}\left(A_{i}\right)=R \geq_{G} R_{r}\left(A_{i}\right)$ so $R_{l}\left(A_{i}\right)^{-1} R_{r}\left(A_{i}\right) \leq_{G} 1$ for all $i . A_{i}$ is positive definite so $W_{i} \leq_{G} 1$ for all $i$. Moreover, we have assumed that for some $i$, inequality is strict: $R_{l}\left(A_{i}\right)^{-1} R_{r}\left(A_{i}\right)<_{G} 1$. By Lemma 3.8, for such $i, W_{i}<_{G} 1$. Thus, $W_{1} \cdots W_{n}<_{G} 1$, which is a contradiction.

As a corollary, we recover a result of Boyer, Gordon and Watson [1; 8].

Corollary 4.2 The fundamental group of the double branched covering of an alternating link is not left-orderable.

Proof Let $D$ be an alternating link diagram. By regarding each crossing of $D$ as a tangle part the elementary tangle $[ \pm 1]$, we get the tangle-strand decomposition of $D$. Since $D$ is alternating, all tangle parts have the same sign. Thus by Theorem 4.1, $\pi_{1}\left(\Sigma_{2}(L)\right)$ is not left-orderable.

As we have mentioned in Remark 1, there are positive definite, non-alternating special algebraic tangles. So links in Theorem 4.1 contain a lot of non-alternating links as well.

\subsection{More non-left-orderable double branched coverings}

Next we give other families of links whose double branched coverings have non-leftorderable fundamental groups. These links are derived from certain quasi-alternating diagrams. See Remark 2 given in Section 5.

Before proving the non-left-orderability, we observe the following rather obvious fact. 
Lemma 4.3 Let $G$ be a group and $A, X, Y \in G$. Assume that $X^{p}=A^{q}$ for some positive integers $p$ and $q$. For a left-ordering $<_{G}$ of $G$, if $1 \leq_{G} Y^{-1} A Y$ (resp. $\left.1 \geq_{G} Y^{-1} A Y\right)$, then $1 \leq_{G} Y^{-1} X Y\left(\right.$ resp. $\left.1 \geq_{G} Y^{-1} X Y\right)$.

Proof $1 \leq_{G}\left(Y^{-1} A Y\right) \leq_{G}\left(Y^{-1} A Y\right)^{q}=Y^{-1} A^{q} Y=Y^{-1} X^{p} Y=\left(Y^{-1} X Y\right)^{p}$, so $1 \leq_{G} Y^{-1} X Y$.

Theorem 4.4 Let $L$ be a link in $S^{3}$ that admits a special tangle-strand decomposition as given in Figure 8 of Example 3. Let $\llbracket m_{i}, M_{i} \rrbracket$ be a universal range of $A_{i}$. Assume that one of the following conditions holds.

(1) $m_{1}, m_{2}, m_{4} \geq 1,-1 \leq m_{3}, m_{5}, m_{6}$ and $M_{3}, M_{5}, M_{6} \leq 0$

(2) $m_{1}, m_{2}, m_{3}, m_{4}, m_{5} \geq 1$ and $A_{6}=Q(r)$ where $-1 \leq r<0$.

Then the fundamental group of the double branched covering $\Sigma_{2}(L)$ is not leftorderable.

Proof Let us consider the coarse Brunner's presentation associated to the tangle-strand decomposition, which we have already given in Example 3. We will frequently use the relation $W_{2} W_{1}=W_{3}$ obtained from the global cycle relations.

Assume that $\pi_{1}\left(\Sigma_{2}(L)\right)$ has a left-ordering $<_{G}$. With no loss of generality, we may assume $A \leq_{G} B$. We will show that all region generators $A, B, C$ are trivial. This contradicts Theorem 3.11, so we conclude $\pi_{1}\left(\Sigma_{2}(L)\right)$ is non-left-orderable.

First we consider the case where the assumption (1) holds.

Case $1 A \leq_{G} B \leq_{G} C$

$B^{-1} A \leq_{G} 1$ so the local coarse relation $W_{4} \in\left[\left[+1,+\infty \rrbracket_{B^{-1} A}\right.\right.$ implies that $W_{4} \leq_{G}$ $B^{-1} A \leq_{G} 1$. Similarly, we get $W_{5} \leq_{G} 1$ and $W_{6} \geq_{G} 1$. Then $W_{3}=W_{5} W_{6}^{-1} \leq_{G} 1$. Since $W_{3} \in\left[-1,0 \rrbracket_{C^{-1}}\right.$, we get $C \leq_{G} 1$ so we conclude $A \leq_{G} B \leq_{G} C \leq_{G} 1$. By the local coarse relation $W_{1} \in[[+1,+\infty]]_{A}, W_{1} A^{-1} \leq_{G} 1$. Then we obtain an inequality

$$
1 \leq_{G} W_{4}^{-1}=W_{1} W_{6} \leq_{G} W_{1}\left(A^{-1} C\right) \leq_{G} W_{1} A^{-1} \leq_{G} 1 .
$$

This implies that all inequalities appearing in the previous arguments must be equalities. This happens only if $A=B=C=1$.

Case $2 A \leq_{G} C \leq_{G} B$

By arguments similar to those in Case 1 , we get $W_{4} \leq_{G} B^{-1} A \leq_{G} 1, W_{5} \geq_{G} 1$ and $1 \leq_{G} W_{6} \leq_{G} A^{-1} C$. Since $W_{5} \in \llbracket-1,0 \rrbracket_{B^{-1} C}, W_{5}\left(B^{-1} C\right) \leq_{G} 1$. Hence

$$
W_{2}=W_{5} W_{4} \leq_{G} W_{5}\left(B^{-1} A\right)=\left[W_{5}\left(B^{-1} C\right)\right]\left(C^{-1} A\right) \leq_{G} W_{5}\left(B^{-1} C\right) \leq_{G} 1 .
$$


By the local coarse relation $W_{2} \in\left[[1,+\infty]_{B}\right.$ and the inequality $W_{2} \leq_{G} 1$, we get $B \leq_{G} 1$. So $A \leq_{G} C \leq_{G} B \leq_{G} 1$. Then as in the Case 1 , we get an inequality

$$
1 \leq_{G} W_{4}^{-1}=W_{1} W_{6} \leq_{G} W_{1}\left(A^{-1} C\right) \leq_{G} W_{1} A^{-1} \leq_{G} 1,
$$

which leads to $A=B=C=1$.

Case $3 C \leq_{G} A \leq_{G} B$

By similar arguments, $W_{4} \leq_{G} 1, W_{5} \geq_{G} 1$ and $W_{6} \leq_{G} 1$. Hence $W_{3}=W_{5} W_{6}^{-1} \geq_{G} 1$. Since $W_{3} \in\left[[-1,0]_{C^{-1}}, 1 \leq_{G} W_{3} \leq_{G} C\right.$, so $1 \leq_{G} C \leq_{G} A \leq_{G} B$. The inequality $1 \leq_{G} A$ and $W_{1} \in\left[[+1,+\infty]_{A}\right.$ implies $W_{1} \geq_{G} 1$. However, this leads to

$$
B \leq_{G} W_{2} \leq_{G} W_{2} W_{1}=W_{3} \leq_{G} C .
$$

Thus, all inequalities appearing in this argument must be equalities. This happens only if $A=B=C=1$.

Next we consider the case the assumption (2) holds.

Case $1 A \leq_{G} B \leq_{G} C$

In this case, $W_{4} \leq_{G} B^{-1} A \leq_{G} 1, W_{5} \geq_{G} 1$ and $W_{6} \geq_{G} 1$. First of all, we determine the sign of $A, B$ and $C$. Assume that $C<_{G} 1$ so $A \leq_{G} B \leq_{G} C<_{G} 1$. Then $W_{1}<_{G} 1$, $W_{2}<_{G} 1, W_{3}>_{G} 1$. This contradicts the relation $W_{3}=W_{2} W_{1}$, so $C \geq_{G} 1$. By a similar argument, we get $A \leq_{G} 1$. Then

$$
W_{2}^{-1}=W_{1} W_{3}^{-1} \geq_{G} W_{1} C=A W_{1} A^{-1} C \geq_{G} A W_{1} W_{6}=A W_{4}^{-1} \geq_{G} A A^{-1} B=B
$$

hence $W_{2}^{-1} \geq_{G} B$. Since $W_{2} \in \llbracket+1,+\infty \rrbracket_{B}$, this implies $B \leq_{G} 1$.

Recall that we have assumed $A_{6}=Q(r)(-1 \leq r<0)$. Let $r=\frac{-q}{p}(p, q \in \mathbb{Z}, \quad p>$ $q>0)$. Then $W_{6}^{-p}=\left(C^{-1} A\right)^{q}$ so $\left(W_{6}^{-1} A^{-1} C\right)^{p}=\left(A^{-1} C\right)^{p-q}$. Since

$$
\left(C^{-1} B\right)^{-1}\left(A^{-1} C\right)\left(C^{-1} B\right)=B^{-1} C A^{-1} B \geq_{G} B^{-1} C \geq_{G} 1,
$$

by applying Lemma 4.3 , we get

$$
\left(C^{-1} B\right)^{-1}\left(W_{6}^{-1} A^{-1} C\right)\left(C^{-1} B\right) \geq_{G} 1 .
$$

Thus

$$
1 \leq_{G}\left(C^{-1} B\right)^{-1} W_{6} C^{-1} B \leq_{G}\left(C^{-1} B\right)^{-1} A^{-1} C\left(C^{-1} B\right)=B^{-1} C A^{-1} B
$$

so we get an inequality $1 \leq_{G} W_{6} C^{-1} B \leq_{G} A^{-1} B$. By obtained inequalities,

$$
W_{1}^{-1} \leq_{G} W_{1}^{-1} W_{2}^{-1}=W_{3}^{-1}=W_{6} W_{5}^{-1} \leq_{G} W_{6} C^{-1} B \leq_{G} A^{-1} B \leq_{G} A^{-1} .
$$


On the other hand, $W_{1} \in[+1,+\infty]_{A}$ implies that $A^{-1} \leq_{G} W_{1}^{-1}$. This implies that all inequalities appearing in this argument must be equalities, so $A=B=C=1$.

Case $2 A \leq_{G} C \leq_{G} B$ or $C \leq_{G} A \leq_{G} B$

If $B<_{G} 1$, then $A, C, B<_{G} 1$ so we get $W_{2}<_{G} 1, W_{1}<_{G} 1$, and $W_{3} \geq_{G} 1$. This contradicts the relation $W_{2} W_{1}=W_{3}$, so $B \geq_{G} 1$. On the other hand, in this case, $W_{4} \leq_{G} 1, W_{5} \leq_{G} 1$ so $W_{2}=W_{5} W_{4} \leq_{G} 1$. However, $W_{2} \in\left[++1,+\infty \rrbracket_{B}\right.$ and $B \geq_{G} 1$ implies that $W_{2} \geq_{G} 1$, so all inequalities above must be equalities. This implies $A=B=C=1$.

Links in Theorem 4.4 contain an interesting family of knots. An oriented knot $K$ is called positive if $K$ is represented by a diagram $D$ having only positive crossings.

Corollary 4.5 Let $K$ be a knot in $S^{3}$ that is positive and genus two. Then the fundamental group of the double branched covering $\Sigma_{2}(K)$ is not left-orderable.

Proof By Corollary 4.2, $\pi_{1}\left(\Sigma_{2}(L)\right)$ is not left-orderable if $L$ is alternating, so we restrict our attention to non-alternating links. Jong and Kishimoto showed that a non-alternating positive knot of genus two is represented by a diagram obtained from three diagrams $9_{39}^{+}, 9_{41}^{+}$and $12_{1202}^{+}$by performing the $\overline{t_{2}^{\prime}}$-moves [9]. See Figure 9 . A knot diagram obtained from the diagram $9_{39}^{+}$belongs to the diagrams in Theorem 4.4 (2), and a knot diagram obtained from the diagram $9_{41}^{+}$and $12_{1202}^{+}$belongs to the diagrams in Theorem 4.4 (1). Thus the double branched coverings of these knots have non-left-orderable fundamental group.
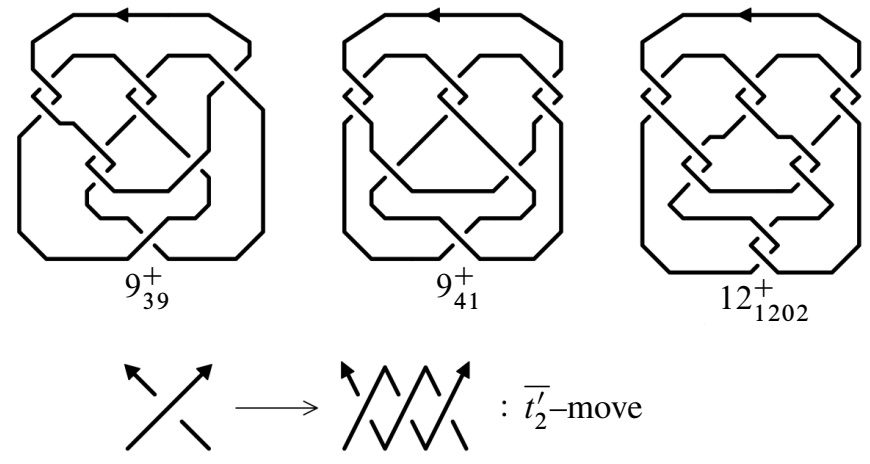

Figure 9: Generators of genus two positive non-alternating knots and $\overline{t_{2}^{\prime}}-$ move

Next we give another example of links having a more complicated tangle-strand decomposition. 
Theorem 4.6 Let $L$ be a link in $S^{3}$ that admits a special tangle-strand decomposition given in Figure 10, and let $\left.\llbracket m_{i}, M_{i} \rrbracket\right]$ be a universal range of $A_{i}$. Assume the following conditions hold.

(1) $A_{1}=Q(r)(\mathbb{Q} \in r, r \geq 1)$ and $A_{4}=A_{10}=Q(-1)$

(2) $m_{2}, m_{3} \geq-1$

(3) $M_{2}, M_{3}, M_{5}, M_{6}, M_{7}, M_{8}, M_{9}<0$

Then $\pi_{1}\left(\Sigma_{2}(L)\right)$ is not left-orderable.
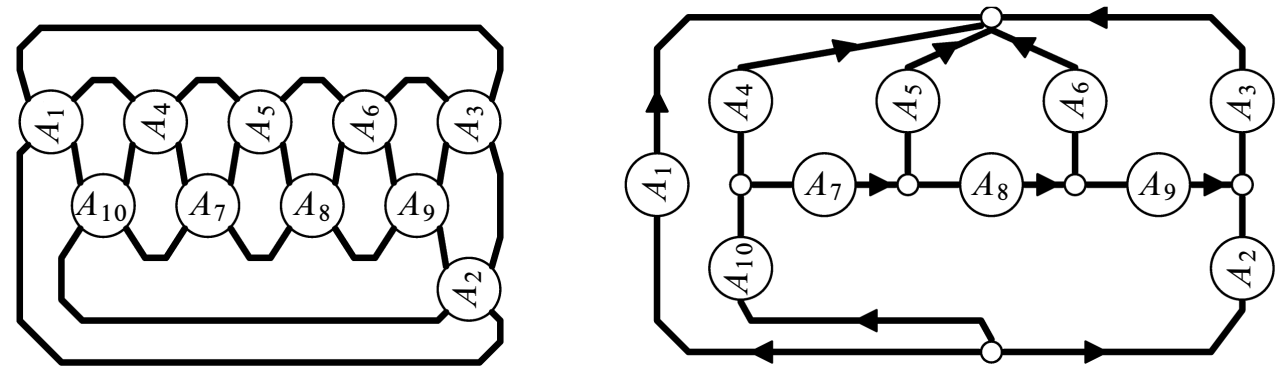

Figure 10: Link diagram and the coarse decomposition graph

Proof The coarse decomposition graph is given on the right in Figure 10. We take region generators $A, B, C, D$ and $E$ as shown on the right in Figure 10 and put $W_{i}=W_{A_{i}}$. First we write down the coarse Brunner's presentation.

\section{[Generators]}

$$
W_{1}, W_{2}, \ldots, W_{10}, A, B, C, D, E
$$

\section{[Local coarse relations]}

$$
\begin{array}{ll}
W_{1} \in \llbracket\left[m_{1}, M_{1} \rrbracket_{A}\right. & W_{2} \in \llbracket\left[m_{2}, M_{2} \rrbracket_{B^{-1}}\right. \\
W_{3} \in \llbracket\left[m_{3}, M_{3} \rrbracket_{E^{-1}}\right. & W_{4} \in \llbracket m_{4}, M_{4} \rrbracket_{A^{-1} C} \\
W_{5} \in \llbracket\left[m_{5}, M_{5} \rrbracket_{C^{-1} D}\right. & W_{6} \in \llbracket m_{6}, M_{6} \rrbracket_{D^{-1} E} \\
W_{7} \in \llbracket\left[m_{7}, M_{7} \rrbracket_{C^{-1} B}\right. & W_{8} \in \llbracket m_{8}, M_{8} \rrbracket_{D^{-1} B} \\
W_{9} \in \llbracket\left[m_{9}, M_{9} \rrbracket_{E^{-1} B}\right. & W_{10} \in \llbracket\left[m_{10}, M_{10} \rrbracket_{A^{-1} B}\right.
\end{array}
$$

[Global cycle relations]

$$
\begin{array}{lll}
W_{1}=W_{4} W_{10}, & W_{9} W_{8} W_{7} W_{10}=W_{2}, & \\
W_{4}=W_{5} W_{7}, & W_{5}=W_{6} W_{8}, & W_{6}=W_{3} W_{9}
\end{array}
$$


Here we remove the trivial region generator to make the presentation simple. By the global cycle relations, $W_{1}=W_{3} W_{2}$ holds, and by assumption (1), $W_{1}^{q}=A^{p}$ $(r=q / p, q>p>0), W_{4}=C^{-1} A$ and $W_{10}=B^{-1} A$.

Assume that $\pi_{1}\left(\Sigma_{2}(L)\right)$ has a left ordering $<_{G}$. With no loss of generality, we may assume $A \leq_{G} B$ holds. As in the proof of Theorem 4.4, we prove non-left-orderability by showing $A=B=C=D=E=1$.

Case $1 B$ is the $<_{G}$-maximal element among the non-trivial region generators $\{A, B, C, D, E\}$.

By the local coarse relations for $W_{7}, \ldots, W_{10}$, we get $W_{7}, W_{8}, W_{9}, W_{10} \leq_{G} 1$. Since $W_{9} W_{8} W_{7} W_{10}=W_{2}, W_{2} \leq_{G} 1$ as well. Since $W_{2} \in[-1,0]_{B^{-1}}, B \leq_{G} 1$. Thus, all region generators $A, B, C, D, E$ are either trivial or $<_{G}-$ negative. By the local coarse relations of $W_{1}$ and $W_{3}$, we obtain $W_{1}, W_{3} \leq_{G} 1$. By the global cycle relations $W_{4}=W_{5} W_{7}, W_{5}=W_{6} W_{8}$ and $W_{6}=W_{3} W_{9}$, we get $W_{4}, W_{5}, W_{6} \leq_{G} 1$. From inequalities $W_{4}, W_{5}, W_{6} \leq_{G} 1$ and their local coarse relations we conclude $A \leq_{G} C \leq_{G} D \leq_{G} E \leq_{G} B \leq_{G} 1$.

Since $A W_{1}^{-1} \geq_{G} 1$ so

$$
1=W_{4} W_{10} W_{1}^{-1}=\left(C^{-1} A\right)\left(B^{-1} A\right)\left(W_{1}^{-1}\right) \geq_{G} C^{-1} A B^{-1} .
$$

Thus, we get $C \geq_{G} A B^{-1}$ and $1 \geq_{G} B C \geq_{G} B A B^{-1}$. As $\left(A^{-1} W_{1}\right)^{q}=A^{p-q}$, Lemma $4.3, B\left(A^{-1} W_{1}\right) B^{-1} \leq_{G} 1$. Hence

$$
W_{1} B^{-1} \leq_{G} A B^{-1} \leq_{G} C .
$$

Finally, we observe

$$
E \leq_{G} W_{3}=W_{1} W_{2}^{-1} \leq_{G} W_{1} B^{-1} \leq_{G} C .
$$

Hence we conclude $C \geq_{G} E$. Since we have already seen $C \leq_{G} E$, this implies that all inequalities appearing in this argument must be equality. This implies $A=B=$ $C=D=E=1$.

Case $2 B$ is not the $<_{G}$-maximal element among the non-trivial region generators $\{A, B, C, D, E\}$.

Since we have assumed $A \leq_{G} B, A$ is not $<_{G}$-maximal. Assume that $C$ is $<_{G}$-maximal. Then $W_{5}, W_{7} \geq_{G} 1$ and $W_{4} \leq_{G} 1$. By the global cycle relation $W_{4}=W_{5} W_{7}$, we conclude these three inequalities must be equalities. This implies $A=B=C=D=E=1$.

Similarly, if $D$ (resp. $E$ ) is $<_{G}$-maximal, then $W_{6}, W_{8} \geq_{G} 1$ and $W_{5} \leq_{G} 1$ (resp. $W_{3}, W_{9} \geq_{G} 1$ and $W_{6} \leq_{G} 1$ ), which leads to the equality $A=B=C=D=E=1$ via the global cycle relation $W_{5}=W_{6} W_{8}$ (resp. $\left.W_{6}=W_{3} W_{9}\right)$. 


\section{Remarks on the L-space conjecture}

We close the paper by giving a remark on the relationships between our work and the L-space conjecture.

It is an interesting problem to study the relationships between orderability of the fundamental groups of a 3-manifold $M$ and its topology or geometry. Boyer, Rolfsen and Wiest showed that if the fundamental group of $M$ is not left-orderable, then $M$ is a rational homology 3-sphere [2].

As for the orderability of the fundamental groups of 3-manifolds, there is a remarkable conjecture:

Conjecture 1 (L-space conjecture [1]) The fundamental group of a rational homology 3-sphere $M$ is non-left-orderable if and only if $M$ is an L-space.

A rational homology 3-sphere $M$ is an $L-$ space if the rank of the Heegaard Floer homology group $\widehat{H F}(M)$ is equal to $\left|H_{1}(M ; \mathbb{Z})\right|$, the cardinal of the 1 st homology group. L-spaces include spherical 3-manifolds, in particular, lens spaces (Ozsváth and Szabó [12]). Recall that we have adopted the convention that the trivial group is not left-orderable, so $S^{3}$ is considered as an L-space with non-left-orderable fundamental group.

This conjecture is verified for Seifert fibered spaces and non-hyperbolic geometric 3-manifolds [1]. There are many examples of 3-manifolds having non-left-orderable fundamental groups (Da̧bkowski, Przytycki and Togha [7], Roberts and Shareshian [15], and Roberts, Shareshian and Stein [16]): many of them are confirmed to be L-spaces (Clay and Watson [6; 5] and Peters [14]). Conversely, many known L-spaces, such as the double branched covering of alternating links, or L-spaces obtained from certain Dehn surgeries are shown to have non-left-orderable fundamental group [1; 8]. (See Corollary 4.2.)

Let us turn to the relationships between our results and the L-space conjecture. A link is called quasi-alternating if it belongs to the set $\mathcal{Q}$ that is the smallest set of links characterized by the following two properties:

(1) The unknot is in $\mathcal{Q}$.

(2) If $L$ has a diagram $D$ with a crossing $c$ such that

(a) the two smoothings $D_{0}$ and $D_{\infty}$ at $c$ (See Figure 11) represent links $L_{0}$, $L_{\infty}$ both of which belong to $\mathcal{Q}$,

(b) $\operatorname{det}\left(L_{0}\right)+\operatorname{det}\left(L_{\infty}\right)=\operatorname{det}(L)$,

then $L$ belongs to $\mathcal{Q}$. Such a crossing $c$ is called a quasi-alternating crossing. 


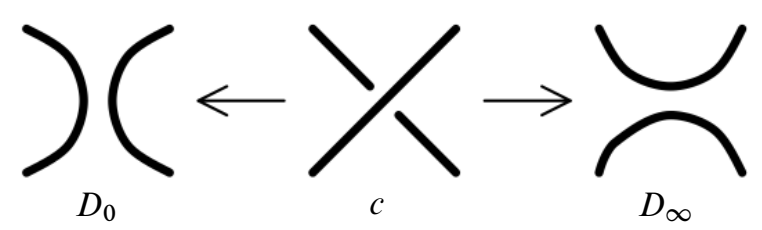

Figure 11: Smoothing $D_{0}$ and $D_{\infty}$

Alternating links are quasi-alternating. In fact, all crossing points of an alternating knot diagram are quasi-alternating crossings. It is known that the double branched covering of a quasi-alternating link $L$ is an $\mathrm{L}$-space [12].

Quasi-alternating links have a nice property with respect to a tangle replacement operation: A link obtained by replacing a quasi-alternating crossing with a rational tangle which extends the crossing is also quasi-alternating (Champanerkar and Kofman [4]). From this property, we can confirm that many links in Theorem 4.1, Theorem 4.4 and Theorem 4.6 are quasi-alternating. So our results provide a lot of new examples of L-spaces with non-left-orderable fundamental groups. For example, the positive knots of genus two are quasi-alternating [9], hence by Corollary 4.5 their double branched coverings are L-spaces having non-left orderable fundamental groups.

On the other hand, we do not know whether all links in our theorems are quasialternating or not, and we do not know whether their double branched coverings $\Sigma_{2}(L)$ are L-spaces or not, either. Thus our family of links also provides a lot of candidates for a counter example to the L-space conjecture.

Remark 2 The diagrams in Theorem 4.4 and Theorem 4.6 are obtained by modifying quasi-alternating, but non-alternating knot diagrams. The diagram in Theorem 4.4 is found by generalizing the diagrams $9_{41}^{+}$and $9_{39}^{+}$in Figure 9, and the diagram in Theorem 4.6 is found by generalizing the quasi-alternating diagram in Ozsváth and Szabó [13, Figure 4].

Using coarse Brunner's presentation arguments, one can find links whose double branched coverings have non-left-orderable fundamental group, by modifying quasialternating diagrams in an appropriate way (that is, by replacing each crossing with a special algebraic tangle having certain universal range). The main point is that the obtained family of links might contain non-quasi-alternating links, so it is unknown whether their double branched coverings are L-spaces or not.

Finally we remark that the coarse presentation method also can be applied to show nonleft-orderability of 3-manifolds obtained as not only the double branched coverings, 
but also as Dehn surgeries, since the well-known Montesinos trick [11] relates some Dehn surgeries and double branched covering constructions.

It is an interesting problem to construct a good coarse presentation from other constructions of 3-manifolds, such as Dehn surgery, general branched coverings, Heegaard splittings and open book decompositions.

\section{References}

[1] S Boyer, C Gordon, L Watson, On L-spaces and left-orderable fundamental groups, to appear in Mathematische Annalen arXiv:1107.5016v1

[2] S Boyer, D Rolfsen, B Wiest, Orderable 3-manifold groups, Ann. Inst. Fourier (Grenoble) 55 (2005) 243-288 MR2141698

[3] A M Brunner, The double cover of $S^{3}$ branched along a link, J. Knot Theory Ramifications 6 (1997) 599-619 MR1468897

[4] A Champanerkar, I Kofman, Twisting quasi-alternating links, Proc. Amer. Math. Soc. 137 (2009) 2451-2458 MR2495282

[5] A Clay, L Watson, On cabled knots, Dehn surgery, and left-orderable fundamental groups, Math. Res. Lett. 18 (2011) 1085-1095 MR2915469

[6] A Clay, L Watson, Left-orderable fundamental groups and Dehn surgery, Int. Math. Res. Not. (2012)

[7] M K Dąbkowski, J H Przytycki, A A Togha, Non-left-orderable 3-manifold groups, Canad. Math. Bull. 48 (2005) 32-40 MR2118761

[8] J Greene, Alternating links and left-orderability arXiv:1107.5232

[9] ID Jong, K Kishimoto, On positive knots of genus two, to appear in Kobe Journal of Mathematics (2011)

[10] L H Kauffman, S Lambropoulou, On the classification of rational tangles, Adv. in Appl. Math. 33 (2004) 199-237 MR2074397

[11] J M Montesinos, Surgery on links and double branched covers of $S^{3}$, from: "Knots, groups, and 3-manifolds (Papers dedicated to the memory of R. H. Fox)", (L P Neuwirth, editor), Ann. of Math. Studies 84, Princeton Univ. Press (1975) 227-259 MR0380802

[12] P Ozsváth, Z Szabó, On knot Floer homology and lens space surgeries, Topology 44 (2005) 1281-1300 MR2168576

[13] P Ozsváth, Z Szabó, On the Heegaard Floer homology of branched double-covers, Adv. Math. 194 (2005) 1-33 MR2141852

[14] T Peters, On L-spaces and non-left orderable 3-manifold groups arXiv: 0903.4495v1 
[15] R Roberts, J Shareshian, Non-right-orderable 3-manifold groups, Canad. Math. Bull. 53 (2010) 706-718 MR2761693

[16] R Roberts, J Shareshian, M Stein, Infinitely many hyperbolic 3-manifolds which contain no Reebless foliation, J. Amer. Math. Soc. 16 (2003) 639-679 MR1969207

Research Institute for Mathematical Sciences, Kyoto University

Sakyo-ku, Kyoto 606-8502, Japan

tetitoh@kurims.kyoto-u.ac.jp

http://www.kurims.kyoto-u.ac.jp/ tetitoh/

Received: 13 April 2012 Revised: 14 February 2013 